\title{
Lactobacillus casei asp23 gene contributes to gentamycin resistance via regulating specific membrane-associated proteins
}

\author{
Wenyi Zhang, ${ }^{*} \dagger$ Chenxia Cao, ${ }^{*} \dagger$ Jie Zhang, ${ }^{*} \dagger$ Lai-Yu Kwok, ${ }^{*} \dagger$ Heping Zhang, ${ }^{*} \dagger$ and Yongfu Chen ${ }^{*}{ }^{1}$ \\ *Key Laboratory of Dairy Biotechnology and Engineering, Ministry of Education, and \\ †Key Laboratory of Dairy Products Processing, Ministry of Agriculture, Inner Mongolia Agricultural University, Hohhot, Inner Mongolia 010018, \\ China
}

\section{ABSTRACT}

Lactobacillus casei Zhang is a probiotic strain originally isolated from koumiss. Previously, we showed that an alkaline shock protein (encoded by asp23) was involved in the adaptation of $L$. casei Zhang to gentamycin. In the present study, we compared the proteomes of the asp23 mutant and its parent strain grown in the presence of gentamycin. The results showed that 22 and 21 proteins were significantly up- and downregulated, respectively ( $>1.5$-fold difference). By parallel reaction monitoring analysis, we further validated that specific membrane-associated proteins were important in regulating the antibiotics-induced cell wall stress. The findings provide insight into the physiological role of the asp23 gene in the growth response of $L$. casei when exposed to antibiotic stress.

Key words: Lactobacillus casei Zhang, proteomics, gentamycin, membrane-associated protein, asp23 gene

\section{INTRODUCTION}

Lactobacillus casei is one of most studied species in the largest lactic acid bacteria genus, Lactobacillus. Members of this species commonly are present in various food environments, such as pickles, cheeses, and yogurt, reflecting their versatility (Bao et al., 2016). The first L. casei genome was decoded in 2009 and has revealed the genomic features contributing to the broad ecological adaptability and evolution of this species (Cai et al., 2009). Particularly, the L. casei species has high flexibility in sugar utilization, as revealed by comparative genomic analysis of several strains, including L. casei Zhang, BL23, and ATCC334 (Zhang et al., 2010). The real breakthrough of the genomic studies on L. casei is driven by the development of highly costeffective and high-throughput DNA sequencing techniques. Using these techniques, we recently investigated

Received October 8, 2017.

Accepted October 31, 2017.

${ }^{1}$ Corresponding author: nmgyfchen@126.com the adaptive evolution of the genomes of $50 \mathrm{~L}$. casei Zhang strains isolated at different stages of prolonged cultivation in an antibiotics-containing environment. This studied probiotic strain was found to have high genome stability even under long-term antibiotic stress (Wang et al., 2017).

Although the genomes of several $L$. case $i$ strains are now available, we are only starting to gain some understanding of the physiology of this species. Further work is required to deepen our knowledge of their cell biology and function. In the last decade, much progress has been made in the study of $L$. casei strain genetics. One classical study performed in $L$. casei BL23 created several genetic mutants of the iolTABCDG1G2EJK cluster. The work deciphered the mechanism of myoinositol utilization of this bacterium (Yebra et al., 2007). With the help of a gene-modifying system derived from a conditionally replicating vector, several interesting genes have been characterized in the same strain (e.g., genes that code for the enzyme L-lactate dehydrogenase, a global catabolite regulator protein, a fibronectin-binding protein, and a 2-component system; Viana et al., 2005; Rico et al., 2008; Muñoz-Provencio et al., 2010; Alcántara et al., 2011). Most of these characterized genes are likely involved in regulating bacterial metabolic capacity or stress response.

The $L$. casei alkaline shock protein, encoded by asp23, is a stress-responsive protein (Zhang et al., 2017). However, its exact cellular function is unknown. Previously, we observed an increase in the sensitivity of gentamycin in the L. casei Zhang asp23 knockout mutant (Zhang et al., 2017). In the present study, we compared the proteomes of the asp23 knockout mutant and its wildtype strain to characterize the biological role of the $L$. casei asp23 gene at protein level.

\section{MATERIALS AND METHODS}

\section{Bacterial Isolates and Culture Conditions}

The asp23 gene disruption mutant and the wild-type strain (L. casei Zhang-G-1200) were obtained from our 
previous studies (Wang et al., 2017; Zhang et al., 2017). The minimum inhibitory concentrations of the wildtype strain and the asp23 mutant were 32 and $16 \mu \mathrm{g} /$ $\mathrm{mL}$, respectively. For proteomic analysis, these bacteria were grown in lactic acid bacteria susceptibility test medium (LSM) supplemented with $8 \mu \mathrm{g} / \mathrm{mL}$ of gentamycin (Klare et al., 2005). At this antibiotic concentration level, the bacterial growth would be inhibited by $50 \%$, as determined by optical density measurement. Growth curves were constructed in relation to optical density, $\mathrm{pH}$, and the number of viable counts based on readings taken every $2 \mathrm{~h}$ during $30 \mathrm{~h}$ of fermentation. All analyses were performed in triplicate.

\section{Sample Preparation}

The asp23 gene disruption mutant and its wild-type strain were collected after $24 \mathrm{~h}$ of cultivation in LSM supplemented with $8 \mu \mathrm{g} / \mathrm{mL}$ of gentamycin. Three biological replicates were prepared for the 2 tested strains. Samples were lysed using SDT lysis buffer (Invitrogen, Carlsbad, CA). Supernatants were collected after centrifuging the samples for $15 \mathrm{~min}$ at $14,000 \times g$ at $25^{\circ} \mathrm{C}$. The Pierce BCA protein assay kit (Thermo Fisher Scientific, Waltham, MA) was used to estimate the sample protein content.

\section{Tandem Mass Tags Labeling and High-pH Reverse- Phase Peptide Fractionation}

Isobaric labels of tandem mass tag (TMT) reagents with varying molecular weights (126-131; Thermo Fisher Scientific) were applied to the digested samples of the wild-type strain and asp23 mutant according to the manufacturer's protocols. Briefly, every sample was labeled with $100 \mu \mathrm{g}$ of TMT tag dissolved in acetonitrile. The labeled peptide mixtures were then fractionated using high-pH reverse-phase chromatography. For each sample, 10 fractions were collected, desalted, and lyophilized.

\section{Liquid Chromatography-MS/MS Analysis}

We performed liquid chromatography (LC)-MS/ MS analysis using the UltiMate 3000 Nano LC System (Thermo Fisher Scientific) coupled to a Q-Exactive hybrid quadrupole-Orbitrap mass spectrometer (Thermo Fisher Scientific) with a nanoelectrospray ionization source. The Orbitrap mass spectrometer was set at a data-dependent mode. Each full MS scan $(60,000$ resolving power) was followed by $6 \mathrm{MS} / \mathrm{MS}$ scans. Based on the scans, the 3 most abundant molecular ions were chosen to be fragmented by collision-induced dissociation (normalized collision energy of $35 \%$ ) before scanning by higher energy collisional dissociation-MS/ MS (collision energy of $45 \%$ ).

\section{Database Search and Quantitative Data Analysis}

The original map file was first generated by TMT quantitative proteomics analysis using the high-resolution Q-Exactive mass spectrometer (Thermo Scientific). Then, the file was converted to the .mgf format by Proteome Discoverer 1.4 software (Thermo Scientific). Data were submitted to the MASCOT2.2 server for database retrieval via built-in software tools (Colaert et al., 2011). Afterward, the library file on the MASCOT server was imported into Proteome Discoverer 1.4 software again. Data screening was then performed with a stringent false discovery rate cut-off value of $<0.01$ to ensure the reliability of the qualitative results.

The protein sequence was qualitatively analyzed by combining the protein databases available for $L$. casei Zhang (Zhang et al., 2010, 2015). Mascot 2.2 was used for database searching and quantitative analysis. Proteome Discoverer 1.4 was used to extract the peptide ion peak intensity, and the quantitative values were normalized. Differential expression of individual proteins between the 2 strains was evaluated with $t$-test (at a cut-off value of an adjusted false discovery rate $P$ value $<0.05)$. All differentially expressed proteins were distributed over clusters of orthologous genes (COG) and were subjected to the Kyoto Encyclopedia of Genes and Genomes database search (Tatusov et al., 1997).

\section{Validation of Protein Expression by Parallel Reaction Monitoring}

To validate the protein expression data obtained by TMT label-free analysis, LC-parallel reaction monitoring (PRM) MS analysis was used to quantify the expression of selected proteins (Peterson et al., 2012). Briefly, peptides were prepared according to the TMT label-free protocol, and an AQUA stable isotope peptide was spiked in each sample as an internal standard reference. Tryptic peptides were desalted on C18 stage tips before being separated by reverse-phase chromatography on an Easy nLC-1200 system (Thermo Scientific). One-hour LC gradients with acetonitrile ranging from 5 to $23 \%$ in 42 min were used. The PRM analysis was carried out with a Q-Exactive Plus mass spectrometer (Thermo Scientific). The optimal parameters such as collision energy, charge state, and retention times were determined experimentally for the most significantly regulated peptides based on conditions developed from high-intensity and confidence-unique peptides of each target protein. The mass spectrometer was set at the 
positive ion mode. The full MS1 scan was acquired with resolution at 60,000 [at a mass:charge ratio $(\mathrm{m} / \mathrm{z})$ of 200 ], an automatic gain control target value of $3.0 \times$ $10^{-6}$, and a maximum ion injection time of $250 \mathrm{~ms}$. Full MS scans were followed by 20 PRM scans at resolution 30,000 (at $m / z 200$ ), an automatic gain control target value of $3.0 \times 10^{-6}$, and a maximum injection time of $200 \mathrm{~ms}$. The targeted peptides were isolated with a 1.6 Thomson (Th) window. Ion activation and dissociation took place in a higher energy collisional dissociation collision cell with normalized collision energy of 27. Skyline, developed by the MacCoss Lab at the University of Washington in Seattle, was used to analyze the raw data (MacLean et al., 2010). The peptide signal intensities detected from unique peptides of the differentially expressed proteins were normalized to the respective sample and to the standard reference.

\section{RESULTS}

\section{Growth Performance of the Wild-Type Strain and asp23 Mutant in the Presence of Gentamycin}

As shown in Figure 1, the wild-type strain grew rapidly in the gentamycin-containing LSM broth contrasting to the asp23 mutant. The cells of the wild-type strain entered into the log phase within $6 \mathrm{~h}$, and cell density reached a high level of $1.07 \times 10^{9} \mathrm{cfu} / \mathrm{mL}$ after $24 \mathrm{~h}$ of cultivation. Consistently, the $\mathrm{pH}$ value of the growth medium decreased faster for the wild-type strain.

\section{Differentially Expressed Proteins in the Wild-Type Strain Compared with the asp23 Mutant}

A total of 43 proteins were differentially expressed in the wild-type strain compared with the asp23 mutant (Table 1). Around one-fourth of the upregulated proteins fell into the COG category of lipid transport and metabolism (27.3\%), and around one-third of the downregulated proteins were involved in the process of carbohydrate transport and metabolism (33.3\%).

The 6 upregulated proteins that were associated with lipid transport and metabolism were acetyl-CoA carboxylase subunit $\beta$ (LCAZH_2069), a biotin carboxylase (LCAZH_2070), a 3-hydroxymyristoyl-ACP dehydratase (LCAZH_2071), a 3-oxoacyl-ACP synthase (LCAZH_2078), a 3-hydroxymyristoyl-ACP dehydratase (LCAZH_2080), and an esterase (LCAZH_2891). In the $L$. casei Zhang genome, the former 5 proteins are located within the same gene cluster that is responsible for the de novo synthesis of fatty acids (Zhang et al., 2010). Similarly, 4 of the downregulated carbohydrate utilization-related proteins were clustered, and they
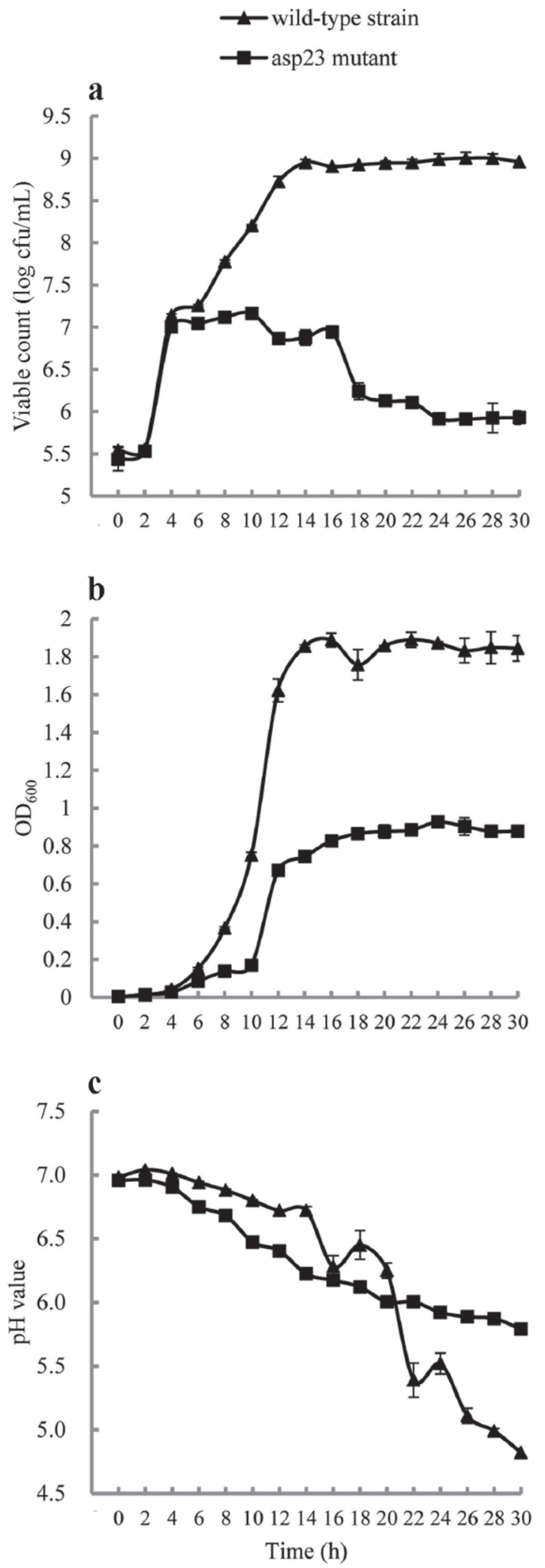

Figure 1. Growth curves of the asp23 mutant and wild-type strain grown in gentamycin-containing lactic acid bacteria susceptibility test medium based on (a) viable counts, (b) optical density (OD) values, and $(\mathrm{c}) \mathrm{pH}$. Error bars represent SD. 
were involved in the sorbitol metabolism (Wang et al., 2016). These proteins included the PTS system sorbitol transporter subunits EIIA (LCAZH_2727), EIIBC (LCAZH_2728), and EIIC (LCAZH_2729) and sorbitol-6-phosphate dehydrogenase (LCAZH_2732). Other differentially expressed proteins belonged to the COG categories of AA transport and metabolism $[\mathrm{E}]$ and inorganic ion transport and metabolism $[\mathrm{P}]$, including tetrahydrodipicolinate $N$-succinyltransferase (LCAZH_0107), the peptidase T (LCAZH_0338), a cation transport ATPase (LCAZH_0107), and a $\mathrm{Mn}^{2+}$ / $\mathrm{Zn}^{2+}$ ABC transporter permease (LCAZH_2388).

\section{Validation of Protein Expression by PRM}

To verify the protein expression data obtained by proteomic analysis, 5 differentially upregulated proteins were selected for further PRM validation. Up to 3 peptides were chosen for each protein for the quantification. As shown in Table 2, the upregulation of the 5 proteins ranged from 2.19- to 5.49-fold, which was consistent with the results obtained from proteomic analysis.

\section{DISCUSSION}

Based on our previous study, an alkaline shock protein (encoded by the asp23 gene) was involved in the adaptation of $L$. casei Zhang to gentamycin. The high expression of this protein in the adapted strain is associated with the development of the resistant phenotype, whereas the deletion of this gene from the genome increases the bacterial sensitivity to gentamycin. Thus, to further investigate its cellular role in coping with gentamycin stress, this work compares the proteomes of the asp23 gene deletion mutant with its parental strain.

Bacterial cell walls act as a physical barrier to protect the cells from external stress (van de Guchte et al., 2002). In particular, membrane alterations play an important role in response to common stresses exerted from the direct vicinity (Coucheney et al., 2005). For instance, osmotic stress, heat shock, or Tween 80 medium supplementation could induce significant modifications in the membrane fatty acid composition of Lactococcus lactis cells (Guillot et al., 2000). The most obvious change was the increase in cyclopropane fatty acid delta C19:0 content while maintaining a stable unsaturated:saturated ratio when the cells were subject to high osmolatity (Guillot et al., 2000). Similarly, when $L$. casei was exposed to an acidic environment, a cyclopropane fatty acid synthase-like methyltransferase was induced and expressed constitutively (Wang et al., 2017). The enzyme catalyzed the addition of a methy- lene residue across the cis double bond of $\mathrm{C} 16: \ln (9)$, C18:1n(9), or C18:1n(11) UFA to form an unsaturated cyclopropane derivative (Wang et al., 2017). Here, we observed the upregulation of 5 proteins in the fatty acid synthetic pathway, among which the acetyl-CoA carboxylase catalyzed the first step of the pathway by carboxylating acetyl-CoA to form malonyl-CoA (Fujita et al., 2007). In the presence of gentamycin, the wild type grew better than the asp23-inactivated mutant, suggesting that the asp23 gene product played a protective role via activating the fatty acid metabolism.

The LCAZH_2891-encoded esterase is another cell wall-associated differentially expressed protein. Although the precise role of this protein in antibiotic resistance is unclear, it is known to be responsive to environmental stress. Our previous proteomic study observed a strong upregulation of an $L$. casei esterase when the cells were subject to acid shock, suggesting that it was likely involved in adaptation to an acidic environment (Wu et al., 2011). Moreover, some esterase homologs were involved in the early response to acid shock and bile stress in Lactobacillus reuteri; the inactivation of the corresponding gene from the bacterium significantly decreased its survival rate under such stress (Wall et al., 2007).

During the late stage of growth, L. casei tends to alternate the carbon catabolic pathway by increasing the utilization of AA and metal ions to sustain proliferation (Wang et al., 2012), which may explain the upregulation of the carbohydrate, AA, and inorganic ion-related proteins in the gentamycin-adapted strain. Interestingly, trehalose-6-phosphate hydrolase, which functions to take up trehalose, was also upregulated. This protein is a major player in trehalose metabolism, which catalyzes the conversion of trehalose-6-phosphate into glucose and glucose-6-phosphate (Termont et al., 2006). The protein has been shown to protect cells from certain environmental stresses (e.g., cryoprotection in L. acidophilus; Duong et al., 2006). However, whether this protein serves a similar role in the case of gentamycin stress still remains to be further explored.

Some sorbitol-related proteins were apparently downregulated in the asp23 gene deletion mutant. These proteins were associated with carbohydrate transport and metabolism. Several proteomic studies found an increase in the expression of this group of protein when the culture shifted from the exponential growth phase to the stationary phase (Laakso et al., 2011; Wang et al., 2016). Our study observed that the adapted strain (wild type) grew better than the asp23 gene deletion mutant in the gentamycin-containing medium; thus, such protein expression changes could be regarded as signatures of phase transition of the asp23 mutant. 
Table 1. Differentially expressed proteins in wild-type strain compared with asp23 mutant in the presence of gentamycin

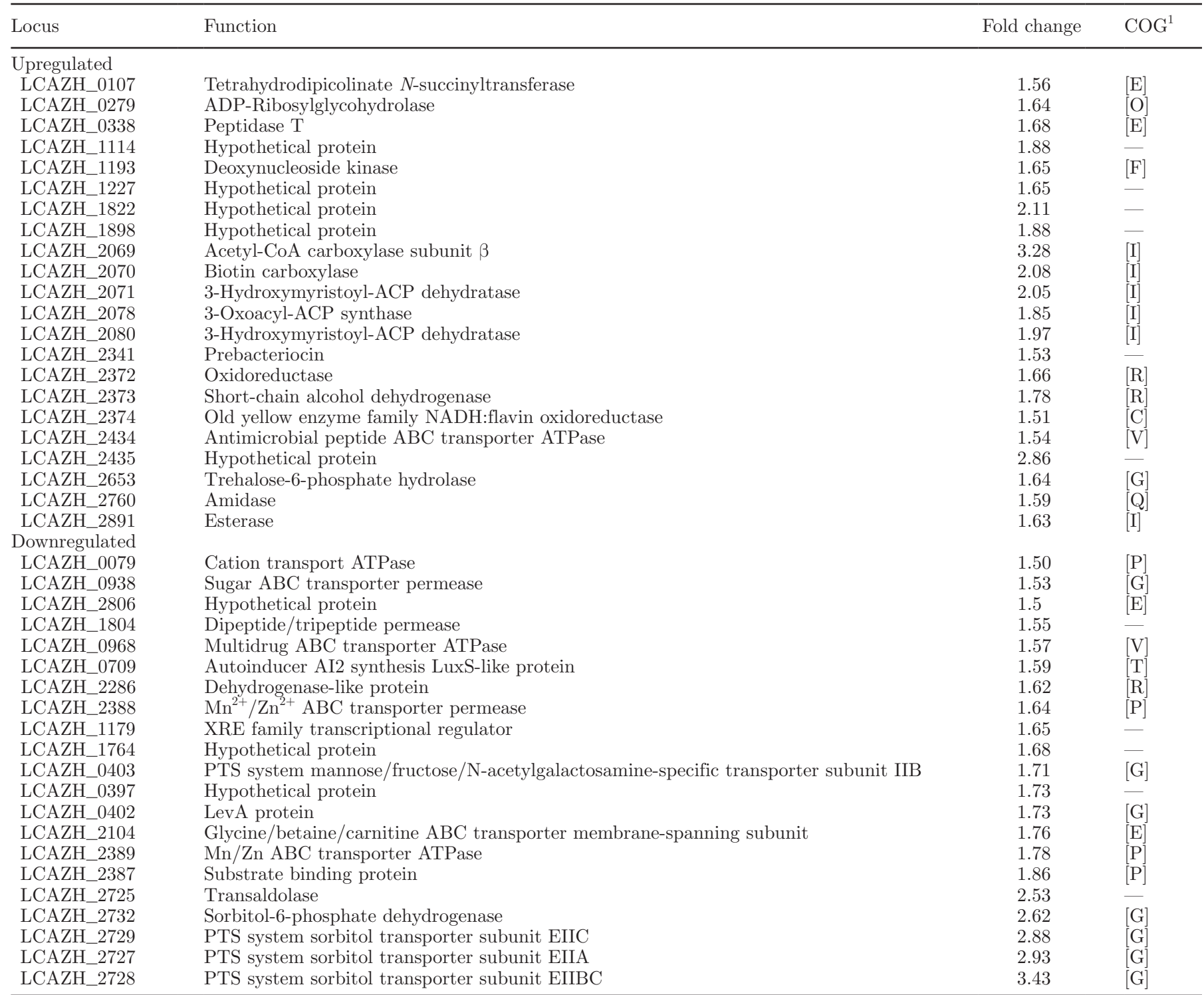

${ }^{1} \mathrm{COG}=$ clusters of orthologous genes. Functional categories: $[\mathrm{C}]=$ energy production and conversion; [E] AA transport and metabolism; [F] $=$ nucleotide transport and metabolism; $[\mathrm{G}]=$ carbohydrate transport and metabolism; $[\mathrm{I}]=$ lipid transport and metabolism; $[\mathrm{O}]=$ posttranslational modification, protein turnover, chaperones; $[\mathrm{P}]=$ inorganic ion transport and metabolism; $[\mathrm{Q}]=$ secondary metabolites biosynthesis, transport and catabolism; $[\mathrm{R}]=$ general function prediction only; $[\mathrm{T}]=$ signal transduction mechanisms; $[\mathrm{V}]=$ defense mechanisms

Table 2. Parallel reaction monitoring validation of the selected upregulated proteins

\begin{tabular}{llc}
\hline Locus & Signature peptides & Ratio \\
\hline LCAZH_2070 & NVVSAAILSGAEAVHPGFGFLSENAEFAK & 5.21 \\
LCAZH_2071 & DFALALFQAPTVLAGK & \\
& TLEAQEIQAILPHR & 2.66 \\
& LIDNAGLGK & \\
LCAZH_2078 & TAYLGGIK & 4.24 \\
& AVYQFAISEVPR & 5.49 \\
LCAZH_2080 & LVDWHDR & 2.19 \\
LCAZH_2891 & YPLFYIDHVSELVPDESLVAEK & \\
& LLPDVDWETEYR & \\
\hline
\end{tabular}




\section{CONCLUSIONS}

In the present study, we compared the proteomes between the asp23 gene deletion mutant and its parent strain grown in gentamycin-containing medium. Interestingly, some membrane-associated proteins were significantly upregulated in the parent strain compared with the asp23 mutant, suggesting that the asp23-encoded alkaline shock protein coped with the gentamycin stress via modulating the cell membrane proteins. Our study has provided insight into the physiological role of asp23 in antibiotic resistance in $L$. casei.

\section{ACKNOWLEDGMENTS}

This research was supported by the Natural Science Foundation of Inner Mongolia (grant no. 2017ZD07; Hohhot) and the Program for Young Talents of Science and Technology in Universities of Inner Mongolia Autonomous Region (grant no. NJYT-17-B05; Hohhot).

\section{REFERENCES}

Alcántara, C., A. Revilla-Guarinos, and M. Zuniga. 2011. Influence of two-component signal transduction systems of Lactobacillus casei BL23 on tolerance to stress conditions. Appl. Environ. Microbiol. 77:1516-1519.

Bao, Q., Y. Song, H. Xu, J. Yu, W. Zhang, B. Menghe, H. Zhang, and Z. Sun. 2016. Multilocus sequence typing of Lactobacillus casei isolates from naturally fermented foods in China and Mongolia. J. Dairy Sci. 99:5202-5213.

Cai, H., R. Thompson, M. F. Budinich, J. R. Broadbent, and J. L. Steele. 2009. Genome sequence and comparative genome analysis of Lactobacillus casei: Insights into their niche-associated evolution. Genome Biol. Evol. 1:239-257.

Colaert, N., H. Barsnes, M. Vaudel, K. Helsens, E. Timmerman, A. Sickmann, K. Gevaert, and L. Martens. 2011. Thermo-msf-parser: An open source Java library to parse and visualize Thermo Proteome Discoverer msf files. J. Proteome Res. 10:3840-3843.

Coucheney, F., L. Gal, L. Beney, J. Lherminier, P. Gervais, and J. Guzzo. 2005. A small HSP, Lo18, interacts with the cell membrane and modulates lipid physical state under heat shock conditions in a lactic acid bacterium. Biochim. Biophys. Acta 1720:92-98.

Duong, T., R. Barrangou, W. M. Russell, and T. R. Klaenhammer 2006. Characterization of the tre locus and analysis of trehalose cryoprotection in Lactobacillus acidophilus NCFM. Appl. Environ. Microbiol. 72:1218-1225.

Fujita, Y., H. Matsuoka, and K. Hirooka. 2007. Regulation of fatty acid metabolism in bacteria. Mol. Microbiol. 66:829-839.

Guillot, A., D. Obis, and M. Y. Mistou. 2000. Fatty acid membrane composition and activation of glycine-betaine transport in Lactococcus lactis subjected to osmotic stress. Int. J. Food Microbiol. $55: 47-51$

Klare, I., C. Konstabel, S. Muller-Bertling, R. Reissbrodt, G. Huys, M. Vancanneyt, J. Swings, H. Goossens, and W. Witte. 2005. Evaluation of new broth media for microdilution antibiotic susceptibility testing of lactobacilli, pediococci, lactococci, and bifidobacteria. Appl. Environ. Microbiol. 71:8982-8986.

Laakso, K., K. Koskenniemi, J. Koponen, M. Kankainen, A. Surakka, T. Salusjarvi, P. Auvinen, K. Savijoki, T. A. Nyman, N. Kalkkinen, S. Tynkkynen, and P. Varmanen. 2011. Growth phase-associated changes in the proteome and transcriptome of Lactobacillus rhamnosus GG in industrial-type whey medium. Microb. Biotechnol. 4:746-766.

MacLean, B., D. M. Tomazela, N. Shulman, M. Chambers, G. L. Finney, B. Frewen, R. Kern, D. L. Tabb, D. C. Liebler, and M. J. MacCoss. 2010. Skyline: An open source document editor for creating and analyzing targeted proteomics experiments. Bioinformatics 26:966-968.

Muñoz-Provencio, D., G. Perez-Martinez, and V. Monedero. 2010. Characterization of a fibronectin-binding protein from Lactobacillus casei BL23. J. Appl. Microbiol. 108:1050-1059.

Peterson, A. C., J. D. Russell, D. J. Bailey, M. S. Westphall, and J. J. Coon. 2012. Parallel reaction monitoring for high resolution and high mass accuracy quantitative, targeted proteomics. Mol. Cell. Proteomics 11:1475-1488.

Rico, J., M. J. Yebra, G. Perez-Martinez, J. Deutscher, and V. Monedero. 2008. Analysis of ldh genes in Lactobacillus casei BL23: Role on lactic acid production. J. Ind. Microbiol. Biotechnol. 35:579-586.

Tatusov, R. L., E. V. Koonin, and D. J. Lipman. 1997. A genomic perspective on protein families. Science 278:631-637.

Termont, S., K. Vandenbroucke, D. Iserentant, S. Neirynck, L. Steidler, E. Remaut, and P. Rottiers. 2006. Intracellular accumulation of trehalose protects Lactococcus lactis from freeze-drying damage and bile toxicity and increases gastric acid resistance. Appl. Environ. Microbiol. 72:7694-7700.

van de Guchte, M., P. Serror, C. Chervaux, T. Smokvina, S. D. Ehrlich, and E. Maguin. 2002. Stress responses in lactic acid bacteria. Antonie van Leeuwenhoek 82:187-216.

Viana, R., G. Perez-Martinez, J. Deutscher, and V. Monedero. 2005. The glycolytic genes pfk and pyk from Lactobacillus casei are induced by sugars transported by the phosphoenolpyruvate:sugar phosphotransferase system and repressed by CcpA. Arch. Microbiol. 183:385-393.

Wall, T., K. Bath, R. A. Britton, H. Jonsson, J. Versalovic, and S. Roos. 2007. The early response to acid shock in Lactobacillus reuteri involves the $C l p \mathrm{~L}$ chaperone and a putative cell wall-altering esterase. Appl. Environ. Microbiol. 73:3924-3935.

Wang, J., X. Dong, Y. Shao, H. Guo, L. Pan, W. Hui, L. Y. Kwok, H. Zhang, and W. Zhang. 2017. Genome adaptive evolution of Lactobacillus casei under long-term antibiotic selection pressures. BMC Genomics 18:320.

Wang, J., W. Hui, C. Cao, R. Jin, C. Ren, H. Zhang, and W. Zhang. 2016. Proteomic analysis of an engineered isolate of Lactobacillus plantarum with enhanced raffinose metabolic capacity. Sci. Rep. 6:31403.

Wang, J. C., W. Y. Zhang, Z. Zhong, A. B. Wei, Q. H. Bao, Y. Zhang, T. S. Sun, A. Postnikoff, H. Meng, and H. P. Zhang. 2012. Transcriptome analysis of probiotic Lactobacillus casei Zhang during fermentation in soymilk. J. Ind. Microbiol. Biotechnol. 39:191-206.

Wu, R., W. Zhang, T. Sun, J. Wu, X. Yue, H. Meng, and H. Zhang. 2011. Proteomic analysis of responses of a new probiotic bacterium Lactobacillus casei Zhang to low acid stress. Int. J. Food Microbiol. 147:181-187.

Yebra, M. J., M. Zuniga, S. Beaufils, G. Perez-Martinez, J. Deutscher, and V. Monedero. 2007. Identification of a gene cluster enabling Lactobacillus casei BL23 to utilize myo-inositol. Appl. Environ. Microbiol. 73:3850-3858.

Zhang, W., H. Guo, C. Cao, L. Li, L. Y. Kwok, H. Zhang, and Z. Sun. 2017. Adaptation to gentamicin in Lactobacillus casei Zhang involves an alkaline shock protein. Front. Microbiol. 8:2316. https:// doi.org/10.3389/fmicb.2017.02316.

Zhang, W., Z. Sun, B. Menghe, and H. Zhang. 2015. Short communication: Single molecule, real-time sequencing technology revealed species- and strain-specific methylation patterns of 2 Lactobacillus strains. J. Dairy Sci. 98:3020-3024.

Zhang, W., D. Yu, Z. Sun, R. Wu, X. Chen, W. Chen, H. Meng, S $\mathrm{Hu}$, and H. Zhang. 2010. Complete genome sequence of Lactobacillus casei Zhang, a new probiotic strain isolated from traditional homemade koumiss in Inner Mongolia, China. J. Bacteriol. 192:5268-5269. 